\title{
RESEÑA DEL LIBRO “EL ARBOLADO EN CIUDADES Y LA PROTECCIÓN DE LOS RECURSOS HÍDRICOS: PREGUNTAS Y RESPUESTAS"
}

\section{Review of the book "Trees in cities and the protection of water resources: questions and answers"}

\section{Elí Misael Bobadilla-Peñaló}

Grupo Sierra Septentrional. Herbario Erik Leonard Ekman (Herbario ELE). Grupo de Investigación Biodiversidad, Ecología y Conservación (Universidad ISA). Grupo Sierra Septentrional Santiago, República Dominicana. ORCID: 0000-0002-0725-9854, Correo-e: ebobadilla@isa.edu.do

Recibido: 22/11/2021 • Aprobado: 13/12/2021

Cómo citar: Bobadilla-Peñaló, E. M. (2021). "El arbolado en ciudades y la protección de los recursos hídricos: preguntas y respuestas". Ciencia, Ambiente y Clima, 4(2), 35-37. Doi: https://doi.org/10.22206/cac.2021.v4i2.pp35-37

El Arbolado en Ciudades y la Protección de los Recursos Hídricos: preguntas y respuestas es un texto de educación ambiental orientado al ciudadano de a pie; una herramienta para que este pueda construir conocimientos teóricos y prácticos, al mismo tiempo que despierta vocaciones por la conservación del arbolado urbano y los servicios ecosistémicos que este ofrece para la seguridad hídrica de la urbe.

El texto, autoría de los profesores-investigadores Solhanlle Bonilla-Duarte, Geralda Díaz, Leonardo Cortés y Ulises Jáuregui-Haza del Instituto Tecnológico de Santo Domingo (INTEC), es el producto principal del proyecto "Servicios ecosistémicos y potencial de adaptación al cambio climático del arbolado urbano del municipio de Santiago de los Caballeros" financiado por el Fondo de Agua del Yaque del Norte y la Fundación Propagas, y llevado a cabo en el período noviembre 2020-noviembre de 2021. Como obra, es un esfuerzo de educación ambiental por llevar a la sociedad un instrumento que le ayude

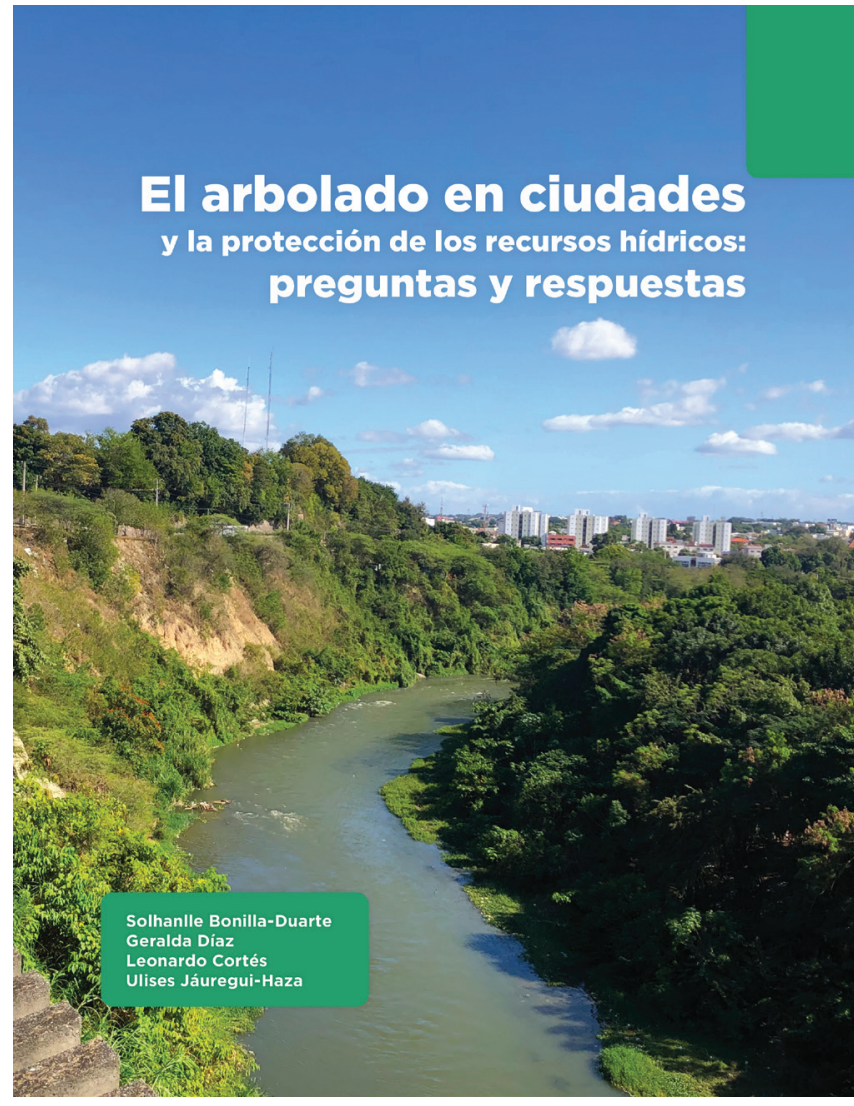


a comprender la importancia del arbolado en la ciudad y cómo estos ofrecen diversos servicios ecosistémicos, esenciales en el mejoramiento de la calidad de vida en la urbe.

El texto se presenta a modo de preguntas y respuestas, facilitando de esta manera la interacción entre los lectores y los autores. En ese sentido, el manual está centrado, fundamentalmente, en dos bloques: el primero sobre contenidos en torno al ecosistema, los servicios ecosistémicos, las especies de árboles más importantes para la urbe y la relación de estos con los recursos hídricos; el segundo, sobre la herramienta $i$-Tree y cómo utilizar esta para el estudio del arbolado urbano y la cuantificación de los servicios ecosistémicos que provee.

La obra es una expresión de la creciente preocupación de la comunidad científica y los ecólogos urbanos sobre el avance de la frontera urbana y cómo las implicaciones de este fenómeno pueden poner en riesgo la seguridad de las personas y afectar la resiliencia climática de las ciudades. Es un punto de partida importante, un complemento en la tarea de concientizar a las personas y crear un modelo de desarrollo basado en el individuo que priorice las buenas prácticas ambientales y la cultura de desarrollo sostenible, en el entendido de las repercusiones que estas pueden tener sobre el colectivo.

Cada una de las preguntas relacionadas a los contenidos del manual son contestadas de forma simple, procurando eliminar el uso excesivo de tecnicismos que son propios del campo de la ecología y la ciencia ambiental, para lograr con ello que el mensaje sea comprensivo y llegue de manera eficiente al público para el que ha sido diseñado. Además, gran parte de los contenidos han sido enriquecidos con ejemplos y enlaces externos a otros recursos utilizando códigos QR, para ampliar los aspectos abordados.

La segunda parte del manual realiza una presentación general de la herramienta $i$-Tree, un software de uso libre utilizado para la cuantificación de los servicios ecosistémicos que ofrece el arbolado en las urbes y cómo esto supone una garantía para el mantenimiento de las condiciones hídricas idóneas. De manera particular, esta segunda parte presenta el conjunto de aplicaciones que posee $i$-Tree y los análisis que se pueden realizar a partir de cada una de ellas, incluidos los requerimientos técnicos de las computadoras a utilizar, los datos de entrada requeridos y la instrumentación necesaria para la recolección de estos, elementos esenciales para el desarrollo de un proyecto. Igualmente, presenta los recursos pedagógicos que se han desarrollado para su uso en la enseñanza en diferentes niveles educativos.

En este apartado, se presentan también los más de 15 proyectos locales que se han llevado a cabo en los últimos años en la cuantificación de los servicios ecosistémicos que ofrecen las áreas verdes urbanas en República Dominicana, que se espera aumente significativamente en las próximas décadas. En este sentido, los retos más importantes serán el establecimiento definitivo y permanente de un sistema de monitoreo y medición de contaminantes aéreos, la instalación de más estaciones meteorológicas en las ciudades capaces de registrar información cada hora del día, la realización de inventarios de árboles más actualizados y en más ciudades, entre otros. Todos estos aspectos son fundamentales para realizar cuantificaciones de los servicios ecosistémicos más eficientes, como el secuestro de dióxido de carbono, por ejemplo. Igualmente se prevé la inclusión de caracteres funcionales de las especies de árboles para obtener datos de una mayor resolución y que sirvan de forma más efectiva a la toma de decisiones para el ordenamiento territorial, la construcción de resiliencia climática urbana y el desarrollo sostenible de las ciudades.

En la parte final de la obra se presentan dos tutoriales sobre la herramienta $i$-Tree: uno sobre la configuración de un proyecto, y otro sobre cómo realizar mediciones utilizando la aplicación i-Tree Canopy. Estos tutoriales representan un aporte importante para 
animar a los ciudadanos en la creación de proyectos, ya sea en los jardines de sus viviendas, sus comunidades, ciudades o espacios urbanos de mayor envergadura. Todas estas opciones son posibles gracias al entramado de aplicaciones que presenta $i$-Tree.

El Arbolado en Ciudades y la Protección de los Recursos Hidricos: preguntas y respuestas es una obra sencilla, pero de gran importancia para la educación ciudadana. Su función principal, al igual que la de la educación ambiental y para el desarrollo sostenible, es la de despertar vocaciones por el medio ambiente y el uso sostenible de los recursos naturales, lo que le enmarca dentro de la filosofía del desarrollo sustentable y la misión global de lograr los objetivos de desarrollo sostenible. 